\title{
FIRST PASSAGE AND RECURRENCE DISTRIBUTIONS
}

\author{
BY
}

T. E. HARRIS

1. Introduction. We consider Markov chains with denumerable states, designated by $0,1,2, \cdots$, and with transition probabilities independent of time. Letting $x_{0}, x_{1}, \cdots$ be the states after $0,1, \cdots$ steps, we define

$$
\mathrm{P}^{(n)}(i, j)=\mathrm{P}\left(x_{n}=j \mid x_{0}=i\right), \quad n=0,1, \cdots,
$$

where $\mathrm{P}(A \mid B)$ stands for the conditional probability of $A$, given $B$. We assume that for each $i$ and $j$ there is an integer $n=n(i, j)$ such that

$$
\mathrm{P}^{(n)}(i, j)>0 \quad \text { for } n=n(i, j) .
$$

Let $N_{i j}$ be the first-passage time from $i$ to $j ; N_{i j}$ is the smallest positive integer $n$ such that $x_{n}=j$, if $x_{0}=i$. If there is no $n$ such that $x_{n}=j$, then $N_{i j}$ $=\infty$. If $j=i$, we speak of the recurrence time for the state $i$.

We shall usually make the assumption

$$
\mathrm{E}\left(N_{i i}\right)<\infty .
$$

If (1.2) holds, then (1.3) (which is true for all $i$ if it is true for any $i$ ) implies the existence of a set of stationary probabilities $\pi_{j}>0$ satisfying

$$
\begin{aligned}
\pi_{j} & =\lim _{n \rightarrow \infty} \frac{1}{n} \sum_{r=0}^{n} \mathrm{P}^{(r)}(i, j), \\
\pi_{j} & =\sum_{r=0}^{\infty} \pi_{r} \mathrm{P}^{(1)}(r, j), \\
\sum_{j=0}^{\infty} \pi_{j} & =1 .
\end{aligned}
$$

See Feller [4, Chap. 15] for the relevant theory.

Let $\theta_{i j}$ be defined as the probability that the state, initially supposed to be $i$, takes on the value $j$ at least once before returning to $i$. The quantities $\theta_{i j}$ turn out to be very useful.

In $\$ 2$ we derive some identities to be used in the sequel. In $\$ 3$ we consider the distribution of the recurrence time $N_{k k}$ under the assumptions (1.2) and (1.3), for "rare" states-i.e., states for which $\pi_{k}$ is small. Since (assuming that there are infinitely many states) no matter how the states are numbered, we must have $\pi_{k} \rightarrow 0$ as $k \rightarrow \infty$, we can speak of the distribution of $N_{k k}$ for large $k$. It is shown that

Received by the editors February 20, 1952. 


$$
\mathrm{P}\left(\pi_{k} \theta_{k 0} N_{k k}>u\right)=\theta_{k 0}\left(e^{-u}+\epsilon_{k}(u)\right),
$$$$
u>0,
$$

where $\epsilon_{k}(u) \rightarrow 0$ as $k \rightarrow \infty$ for each fixed $u>0$.

In $\S 4$ we give explicit expressions for the $\pi_{j}, \theta_{i j}$, and for mean recurrence and first-passage times, in the case where the Markov chain is a random walk; that is, $\mathrm{P}_{i, i+1}^{(1)}=p_{i}, \mathrm{P}_{i, i-1}^{(1)}=1-p_{i}$. The method depends on the representation of a random walk as a Brownian particle moving among suitably selected points. $\$ 5$ gives a more precise form of Lemma 3 for random walks and a method for getting moments of first-passage times in random walks. §6 gives a rather curious correspondence between random walks and trees.

The author was in correspondence with Professors Chung and Feller while this paper was being written, and both of them furnished alternative proofs of some of the results of $\$ 4$. Some of the identities in $\$ 2$ are closely related to recent and current work of Chung [2], who, in particular, has a result involving three states of which Lemma 1 of $\$ 2$ is a special case. According to Chung, Lemma 2 appears in a work by Paul Lévy [8] which the author has not yet seen.

Dr. Chung was courteous enough to delay publication of his paper until the present paper was ready.

\section{Some identities.}

Lemma $1\left({ }^{1}\right)$. Under the assumptions (1.2) and (1.3), we have

$$
\mathrm{E}\left(N_{i j}+N_{j i}\right)=\frac{1}{\pi_{i} \theta_{i j}},
$$

To prove (2.1), suppose the initial state is $i$, and let $N_{i i}^{(1)}, N_{i i}^{(2)}, \cdots$ be the time intervals between successive recurrences to $i$. Let the (random) integer $R$ designate the first cycle from $i$ back to $i$ during which the state $j$ is visited at least once. Then $N_{i i}^{(1)}+\cdots+N_{i i}^{(R)}$ is a sample value of $N_{i j}+N_{j i}$. Since $\mathrm{E}\left(N_{i i}\right)=1 / \pi_{i}$ and $\mathrm{E}(R)=1 / \theta_{i j}$, we have, making use of a slight modification of a theorem of Wald [10, p. 52]

$$
\mathrm{E}\left(N_{i j}+N_{j i}\right)=\mathrm{E}\left(N_{i i}^{(1)}+\cdots+N_{i i}^{(R)}\right)=\mathrm{E}\left(N_{i i}\right) \mathrm{E}(R)=\frac{1}{\pi_{i} \theta_{i j}},
$$

which proves (2.1)

Since the left side of (2.1) is unchanged if $i$ and $j$ are interchanged, the right side must be also. This leads to the identity

$$
\pi_{j} / \pi_{i}=\theta_{i j} / \theta_{j i} .
$$

Formula (2.3) is useful in the treatment of random walks, since the $\theta_{i j}$ are easy to find and thus the $\pi_{j}$ can be obtained.

The identity (2.3) holds even if $\mathrm{E}\left(N_{i i}\right)=\infty$, provided the chain is recur-

(1) See the next-to-last paragraph of the introduction. 
rent-i.e., $\mathrm{P}\left(N_{i i}<\infty\right)=1$-and provided (1.2) holds. In this case the quantities $\pi_{j} / \pi_{i}$ are Doeblin's ratios (see [3]),

$$
\pi_{j} / \pi_{i}=\lim _{n \rightarrow \infty} \frac{\sum_{r=0}^{n} \mathrm{P}^{(r)}(k, j)}{\sum_{r=0}^{n} \mathrm{P}^{(r)}(l, i)} .
$$

The proof in this case follows from results of Chung in [2] and will not be given here.

As an example of the use of (2.3), we have

Lemma 2(1). (Paul Lévy.) Let $Y_{n}(i)$ be the number of times the state is $i$ during the first $n$ steps from an arbitrary starting point. Then if (1.2) holds and if the chain is recurrent, we have

$$
\lim _{n \rightarrow \infty} Y_{n}(j) / Y_{n}(i)=\pi_{j} / \pi_{i}, \quad i \neq j,
$$

with probability 1.

To prove (2.4), which is trivial if (1.3) holds, we suppose for simplicity that $x_{0}=i$; this does not essentially affect the argument. From the definition of $\theta_{i j}$ it follows that the probability that the state takes on the value $j$ exactly $r$ times between successive visits to $i$ is given by $1-\theta_{i j}$ if $r=0$ and $\theta_{i j}\left(1-\theta_{j i}\right)^{r-1} \theta_{j i}$ if $r>0$. The expected number of visits to $j$ between visits to $i$ is thus $\theta_{i j} / \theta_{j i}$. From the strong law of large numbers it follows that if $n_{1}, n_{2}, \cdots$ are the times at which $x_{n}=i$, we have

$$
\lim _{k \rightarrow \infty} Y_{n_{k}}(j) / Y_{n_{k}}(i)=\theta_{i j} / \theta_{j i} \text {. }
$$

Similar reasoning shows that the same limit is approached if $n$ passes through the sequence of values $\left(n_{k}^{\prime}\right)$ for which $x_{n}=j$. But since the sequences $\left(n_{k}\right)$ and $\left(n_{k}^{\prime}\right)$ are the only values of $n$ for which the ratio $Y_{n}(j) / Y_{n}(i)$ can change, the limit must exist, and use of (2.3) gives (2.4).

3. Distribution of $N_{k k}$ for large $k$. We assume throughout this section that (1.2) and (1.3) hold and that there are infinitely many states; otherwise the Markov chain is arbitrary.

We first consider the distribution of $N_{0 k}$ as $k \rightarrow \infty$, where 0 is an arbitrary fixed state.

Lemma 3. $\operatorname{Lim}_{k \rightarrow \infty} \mathrm{P}\left(\pi_{0} \theta_{0 k} N_{0 k}>u\right)=e^{-u}, u>0$.

Let $u$ denote an arbitrary fixed positive number and denote by $[z]$ the largest integer not greater than $z$. Suppose the initial state is 0 and let $S_{k}$ be the number of steps in the first $\left[u / \theta_{0 k}\right]$ recurrences to 0 . Let $A_{k}$ be the event 
that $k$ is not visited during the first $\left[u / \theta_{0 k}\right]$ recurrences to 0 , and $B_{k}(\epsilon), \epsilon>0$, the event

$$
\left|S_{k} /\left[u / \theta_{0 k}\right]-\frac{1}{\pi_{0}}\right|<\epsilon,
$$

and let $C_{k}$ be the event

$$
N_{0 k}>\left(\frac{1}{\pi_{0}}-\epsilon\right)\left[u / \theta_{0 k}\right] .
$$

Then we have, letting $\bar{B}$ denote the complement of $B$,

$$
\mathrm{P}\left(C_{k}\right) \geqq \mathrm{P}\left(A_{k}, B_{k}\right)=\mathrm{P}\left(A_{k}\right)-\mathrm{P}\left(A_{k}, \bar{B}_{k}\right) \text {. }
$$

Now $\mathrm{P}\left(A_{k}\right)=\left(1-\theta_{0 k}\right)^{\left[u / \theta_{0 k}\right]} \rightarrow e^{-u}$ as $k \rightarrow \infty$, and $\mathrm{P}\left(\bar{B}_{k}\right) \rightarrow 0$, by the law of large numbers, both these limits holding uniformly on any finite $u$-interval bounded away from 0 . An easy consequence of (3.0) is that

$$
\lim _{u \rightarrow \infty} \mathrm{P}\left(\pi_{0} \theta_{0 k} N_{0 k}>u\right) \geqq e^{-u}, \quad u>0 .
$$

A similar argument shows lim sup $\leqq e^{-u}$, and Lemma 3 follows.

A consequence of Lemma 3 is that

$$
\underset{k \rightarrow \infty}{\lim \inf } \mathrm{E}\left(\pi_{0} \theta_{0 k} N_{0 k}\right) \geqq 1 \text {. }
$$

On the other hand, from (2.1) we have

$$
\mathrm{E}\left(\pi_{0} \theta_{0 k} N_{0 k}\right)+\mathrm{E}\left(\pi_{0} \theta_{0 k} N_{k 0}\right)=1 .
$$

From (3.1), (3.2), and (2.3), we have, recalling from (2.3) that $\pi_{0} \theta_{0 k}=\pi_{k} \theta_{k 0}$,

$$
\begin{gathered}
\lim _{k \rightarrow \infty} \mathrm{E}\left(\pi_{0} \theta_{0 k} N_{0 k}\right)=\lim _{k \rightarrow \infty} \mathrm{E}\left(\pi_{k} \theta_{k 0} N_{0 k}\right)=1, \\
\lim _{k \rightarrow \infty} \mathrm{E}\left(N_{k 0}\right) / \mathrm{E}\left(N_{0 k}\right)=0 .
\end{gathered}
$$

We now prove

THEOREM 1. For each $u>0$,

$$
\mathrm{P}\left(\pi_{k} \theta_{k 0} N_{k k}>u\right)=\theta_{k 0}\left(e^{-u}+\epsilon_{k}(u)\right)
$$

where $\epsilon_{k}(u) \rightarrow 0$ as $k \rightarrow \infty$.

The symbols in (3.5) are defined in the introduction. The proof will bring out the intuitive meaning of (3.5). Of course, any fixed state rather than 0 could be used.

Proof. Let $T_{k}$ be the expected value of $N_{k k}$, given that the path from $k$ back to $k$ is never in 0 and let $U_{k}$ be the expected value of $N_{k 0}$ given that 
the path from $k$ to 0 is never in $k$ after the initial position. Now consider a sample first-passage from $k$ to 0 . There will first be $r$ returns to $k, r=0,1, \cdots$, before 0 is visited, and finally a passage from $k$ to 0 during which $k$ is not revisited. Since $r$ has the frequency function $\theta_{k 0}\left(1-\theta_{k 0}\right)^{r}$, we have

$$
\begin{aligned}
\mathrm{E}\left(N_{k 0}\right) & =T_{k} \sum_{r=0}^{\infty} r \theta_{k 0}\left(1-\theta_{k 0}\right)^{r}+U_{k} \\
& =T_{k}\left(\frac{1}{\theta_{k 0}}-1\right)+U_{k} .
\end{aligned}
$$

Now, supposing again that the initial state is $k$, let $D_{k}$ be a random variable which is 1 if the state recurs to $k$ without being in 0 , and 0 otherwise. Then for any $u>0$ we have

$$
\begin{aligned}
\mathrm{P}\left(\pi_{k} \theta_{k 0} N_{k k}>u\right)= & \left(1-\theta_{k 0}\right) \mathrm{P}\left(\pi_{k} \theta_{k 0} N_{k k}>u \mid D_{k}=1\right) \\
& +\theta_{k 0} \mathrm{P}\left(\pi_{k} \theta_{k 0} N_{k 0}+\pi_{k} \theta_{k 0} N_{0 k}>u \mid D_{k}=0\right) .
\end{aligned}
$$

Now $\mathrm{E}\left(N_{k 0} \mid D_{k}=0\right)=U_{k}$, and from (3.6) and (3.4), $U_{k} \leqq \mathrm{E}\left(N_{k 0}\right)=o\left(1 / \pi_{k} \theta_{k 0}\right)$, $k \rightarrow \infty$. Therefore, under the hypothesis $D_{k}=0$, the random variable $\pi_{k} \theta_{k 0} N_{k 0}$ converges in distribution to 0 . Thus, the second term on the right side of (3.7) is, using Lemma $3, \theta_{k 0} e^{-u}\left(1+\bar{\epsilon}_{k}(u)\right)$, where $\bar{\epsilon}_{k}(u) \rightarrow 0$ as $k \rightarrow \infty$.

Since for any non-negative random variable $z$ we have $\mathrm{P}(z>u) \leqq \mathrm{E}(z) / u$, $u>0$, it follows that the first term on the right side of (3.7) is bounded by (using (3.6))

$$
\begin{aligned}
\left(\frac{1-\theta_{k 0}}{u}\right) \pi_{k} \theta_{k 0} \mathrm{E}\left(N_{k k} \mid D_{k}=1\right) & =\frac{\pi_{k} \theta_{k 0}^{2}}{u}\left(\frac{1}{\theta_{k 0}}-1\right) T_{k} \\
& =\frac{\pi_{k} \theta_{k 0}^{2}}{u}\left[\mathrm{E}\left(N_{k 0}\right)-U_{k}\right] \\
& \leqq \frac{\theta_{k 0}}{u} \pi_{k} \theta_{k 0} \mathrm{E}\left(N_{k 0}\right) .
\end{aligned}
$$

Since from (3.4) we have $\pi_{k} \theta_{k 0} \mathrm{E}\left(N_{k 0}\right) \rightarrow 0$ as $k \rightarrow \infty$, Theorem 1 now follows. As we shall see in the next section, the limit inferior of $\theta_{k 0}$ may be 0 as $k$ $\rightarrow \infty$. In this case Theorem 1 is rather empty of content.

4. Random walks. In the remainder of this paper we consider random walks on the integers with transition probabilities $p_{r}$ for $r \rightarrow r+1$ and $q_{r}=1-p_{r}$ for $r \rightarrow r-1$. Some of the results can obviously be extended to the case where the transition $r \rightarrow r$ has a positive probability $\left({ }^{2}\right)$.

During the remainder of this paper we shall assume that one of the two

(2) Added in proof: I have learned in a letter from Mr. F. G. Foster that recent independent work of his overlaps considerably with the work of this section. 
following conditions holds:

Condition A:

$$
0<p_{r}<1, \quad r=1,2, \cdots .
$$

Condition $\mathrm{B}(\omega)$ : there is an integer $\omega>1$ such that

$$
0<p_{r}<1, \quad r=1,2, \cdots, \omega-1 ; p_{\omega}=0 .
$$

Some of the results holding under condition $\mathrm{B}$ will obviously be true whether or not $p_{\omega}=0$. When condition B applies, it is tacitly assumed that all states mentioned have indices not greater than $\omega$.

We make the following definitions. Let

$$
\begin{aligned}
L_{1} & =1, \quad L_{r}=\prod_{j=1}^{r-1}\left(q_{j} / p_{j}\right), \\
z_{0} & =0, \quad z_{r}=\sum_{j=1}^{r} L_{j}, \\
Z & =\sum_{j=1}^{\infty} L_{j} \leqq \infty \text { (applicable when condition A holds) }
\end{aligned}
$$

TheOREM 2a. Suppose condition A holds and let the initial state be $k>0$. The probability that the state never reaches 0 is $z_{k} / Z$.

If $Z=\infty, z_{k} / Z$ is taken to be 0 .

TheOREm 2b. Suppose condition $\mathrm{B}(\omega)$ holds and let the initial state be $k$, $0<k \leqq \omega$. The probability that the state reaches $\omega$ before it reaches 0 is $z_{k} / z_{\omega}$.

Proof of Theorem 2a. Let the points $0=z_{0}, z_{1}, z_{2}, \cdots$ be marked off on a line and suppose that a one-dimensional Brownian motion (Wiener process) takes place on the line. As the Brownian particle moves about let it always bear as a label the subscript of that one of the $z_{r}$ which it has most recently visited. The label then executes a random walk. Using the well known fact that a Brownian particle initially at a point $O$ between points $A$ and $B$ has probability $O B / A B$ of reaching $A$ before $B$, we can verify that the random walk executed by the label has the same transition probabilities $p_{r}, q_{r}$ as the original random walk.

Suppose first that $Z=\lim _{r \rightarrow \infty} z_{r}=\infty$. Since the graph of the position of the particle is continuous, every path from $z_{k}$ to $z_{0}$ corresponds to a definite finite sequence of labels-i.e., a definite walk from $k$ to 0 . Conversely, every walk from $k$ to 0 corresponds to a family of Brownian paths from $z_{k}$ to $z_{0}$, families corresponding to distinct walks being mutually exclusive. The probability of a given walk is the same as the probability of the corresponding Brownian family. Since almost all Brownian paths from $z_{k}$ reach $z_{0}$, almost all random walks from $k$ reach 0 , Q.E.D. 
If $Z<\infty$, walks from $k$ which reach 0 correspond to Brownian paths from $z_{k}$ which reach 0 before reaching $Z$, the probability of these being $1-z_{k} / Z$, Q.E.D.

The proof of Theorem $2 \mathrm{~b}$ is essentially the same.

Using Theorem 2, we can now evaluate the quantities $\theta_{i j}$ for random walks. (We recall that $\theta_{i j}$ is the probability that the state, initially at $i$, reaches $j$ at least once before returning to $i$.) In fact, if $i<j$, we have

$$
\theta_{i j}=\frac{p_{i}}{1+\frac{q_{i+1}}{p_{i+1}}+\frac{q_{i+1} q_{i+2}}{p_{i+1} p_{i+2}}+\cdots+\frac{q_{i+1} \cdots q_{j-1}}{p_{i+1} \cdots p_{j-1}}}, \quad i<j,
$$

and a similar expression holds for $i>j$. If $i=j-1$, the expression on the right of (4.2) is taken as $p_{i}$.

Using (4.2) and (2.3), we can evaluate the stationary probabilities for random walks with finite mean recurrence times (or the Doeblin ratios for recurrent walks with infinite mean times). For example, putting $i=0, j>0$, we have from (2.3), (4.2), and the analogue of (4.2) for the case $j<i$,

$$
\pi_{j}=\pi_{0} \theta_{0 j} / \theta_{j 0}
$$

$$
\begin{aligned}
& =\frac{\pi_{0} p_{0}}{\left(1+\frac{q_{1}}{p_{1}}+\cdots+\frac{q_{1} \cdots q_{j-1}}{p_{1} \cdots p_{j-1}}\right)} \cdot \frac{\left(1+\frac{q_{1}}{p_{1}}+\cdots+\frac{q_{1} \cdots q_{j-1}}{p_{1} \cdots p_{j-1}}\right)}{\frac{q_{1} \cdots q_{j-1}}{p_{1} \cdots p_{j-1}} q_{j}} \\
& =\frac{\pi_{0} p_{0}}{q_{j} L_{j}},
\end{aligned}
$$

where $L_{j}$ is defined by (4.1). A similar expression holds for $j<0$. In the case of finite mean recurrence times the condition $\sum \pi_{j}=1$ completes the determination in (4.3).

As an example of the use of Theorem 2 we have the following corollary.

Corollary. According as A or $\mathrm{B}(\omega)$ holds we have

$$
\begin{aligned}
\mathrm{A} & \rightarrow \mathrm{E}\left(N_{10}\right)=\sum_{j=1}^{\infty} \frac{1}{q_{j} L_{j}}, \\
\mathrm{~B}(\omega) \rightarrow \mathrm{E}\left(N_{10}\right) & =\sum_{j=1}^{\omega} \frac{1}{q_{j} L_{j}} .
\end{aligned}
$$

For we can place a reflecting barrier at 0 , giving $p_{0}=1$. Then $\mathrm{E}\left(N_{10}\right)$ - $\mathrm{E}\left(N_{00}\right)-1=1 / \pi_{0}-1$. (If $\mathrm{B}(\omega)$ holds, $q_{\omega}=1$.)

Since for every $i<j$ we have 


$$
N_{i j}=N_{i, i+1}+N_{i+1, i+2}+\cdots+N_{j-1, j},
$$

it is clear how from Theorem 2 any desired mean recurrence or first-passage time can be found.

As mentioned in the introduction, Professors Chung and Feller have given alternate approaches (correspondence).

It is interesting now to consider random walks of the transient type. We shall see that there are nonrecurrent random walks where the conditional mean recurrence time, given that recurrence takes place, is finite $\left({ }^{3}\right)$. We pick $N_{10}$ as a typical first-passage random variable.

Theorem 3. Suppose condition A holds. The conditional expectation of $N_{10}$, given that $N_{10}<\infty$, is

$$
\mathrm{E}\left(N_{10} \mid N_{10}<\infty\right)=1+\left(\frac{2}{1-1 / Z}\right) \sum_{r=1}^{\infty} \frac{\left(1-z_{r} / Z\right)\left(1-z_{r+1} / Z\right)}{L_{r+1}}
$$

where $z_{r}$ and $L_{r}$ are defined in (4.1), and $Z=\sum_{r=1}^{\infty} z_{r}$.

Before proving Theorem 3 , it is interesting to consider a special example. Suppose for simplicity that $p_{0}=1$ and that the quantities $p_{r}$ have the form

$$
p_{r}=\frac{1}{2}+\frac{c}{r}+O\left(\frac{1}{r^{2}}\right),
$$$$
r \rightarrow+\infty,
$$

where $c$ is independent of $r$. From Theorems 2 and 3 we see that if $c<-1 / 4$, the mean recurrence times are finite; if $-1 / 4 \leqq c \leqq 1 / 4$, the mean recurrence times are infinite but the states are recurrent; if $c>1 / 4$, the recurrence probabilities are less than 1 . In the latter case $\mathrm{E}\left(N_{10} \mid N_{10}<\infty\right)$ is finite or infinite according as $c>3 / 4$ or $1 / 4<c \leqq 3 / 4$. The intuitive significance of this last fact is that if $c$ is large (i.e., $c>3 / 4$ ), then a path from 1 is very unlikely to get back to 0 unless it does so quickly.

To prove Theorem 3 it is convenient to return to the Brownian motion scheme. If $Z=\infty$, then Theorem 3 reduces to the corollary to Theorem 2 (note the identity $\sum_{r=1}^{\infty} 1 / q_{r} L_{r}=\sum_{r=1}^{\infty}\left(p_{r}+q_{r}\right) / q_{r} L_{r}=\sum_{r=1}^{\infty} 1 / L_{r+1}$ $\left.+\sum_{r=1}^{\infty} 1 / L_{r}\right)$ so we may as well take the case $Z<\infty ; Z$ is of course greater than 1. We note that if a Brownian particle is initially at $z_{1}=1$, the conditional probability density for the maximum displacement $y$ attained by the particle before it arrives at 0 , given that $y<Z$, is $1 /\left[(1-1 / Z) y^{2}\right]$.

Consider a Brownian path starting at $z_{1}=1$, and let $M(y)$ be the expected number of label changes before reaching $y>z_{1}$, given that $y$ is reached before $z_{0}=0$. (We shall always suppose that $y$ is not one of the points $z_{r}$.) It is intuitively obvious (we omit the formal proof, which is not difficult) that the conditional expectation of the number of label changes in going from $y$ back

(3) This can also be seen from results of Chung [2]. 
to $z_{0}$, given that $y$ is the maximum displacement, is $M(y)+1$, the added 1 being for the change which occurs when $z_{0}$ is reached. Therefore the total conditional expectation in a path from $z_{1}$ to $z_{0}$, given that $y$ is the maximum displacement, is $1+2 M(y)$. Thus

$$
\mathrm{E}\left(N_{10} \mid N_{10}<\infty\right)=1+\frac{2}{1-1 / Z} \int_{1}^{Z} \frac{M(y) d y}{y^{2}} .
$$

Clearly $M(y)=0$ for $z_{1}<y<z_{2}$. To evaluate $M(y)$ for $y>z_{2}$, suppose that $z_{k}$ is the rightmost of the points $z_{1}, z_{2}, \cdots$ lying to the left of $y$, and consider a Brownian path from $z_{1}$ to $y$ which reaches $y$ before $z_{0}$. Let $R_{j}, 1 \leqq j \leqq k-1$, be the total number of label changes which this path would undergo if only $z_{j}$ and $z_{j+1}$, and no other points of the set $z_{1}, z_{2}, \cdots$, were present, and if the label were initially taken to be $j$. The total number of changes is then $R_{1}$ $+R_{2}+\cdots+R_{k-1}$ (it helps to draw a diagram); hence

$$
M(y)=\sum_{j=1}^{k-1} \mathrm{E}\left(R_{j} \mid D\right)
$$

where $D$ is the event that the path starting from $z_{1}$ reaches $y$ before 0 . Now $\mathrm{E}\left(R_{j} \mid D\right)$ can be found by elementary combinatorial means since, translated back in terms of random walks, a random walk with only four states is involved. In fact

$$
\mathrm{E}\left(R_{j} \mid D\right)=\frac{z_{j}+z_{j+1}-2 z_{j} z_{j+1} / y}{z_{j+1}-z_{j}} .
$$

From (4.5), (4.6), and (4.7) we have

$$
\begin{aligned}
\mathrm{E}\left(N_{10} \mid N_{10}<\infty\right) \\
=1+\left(\frac{2}{1-1 / Z}\right) \lim _{N \rightarrow \infty} \sum_{k=1}^{N} \int_{z k}^{z z_{k+1}} \sum_{j=1}^{k-1}\left(\frac{z_{j}+z_{j+1}-2 z_{j} z_{j+1} / y}{z_{j+1}-z_{j}}\right) \frac{d \nu}{y^{2}} \\
=1+\left(\frac{2}{1-1 / Z}\right) \lim _{N \rightarrow \infty} \sum_{j=1}^{N-1} \frac{\left(1-z_{j} / z_{N+1}\right)\left(1-z_{j+1} / z_{N+1}\right)}{z_{j+1}-z_{j}} \\
=1+\left(\frac{2}{1-1 / Z}\right) \sum_{j=1}^{\infty} \frac{\left(1-z_{j} / Z\right)\left(1-z_{j+1} / Z\right)}{z_{j+1}-z_{j}} .
\end{aligned}
$$

The passage to limit under the summation sign is justified because each term in the sum is positive and increases with $N$. This gives Theorem 3.

If condition $\mathrm{B}(\omega)$ holds, then we have the following analogue of Theorem 3 , proof of which is entirely similar:

The expected value of $N_{10}$, given that 0 is reached before $\omega, i s$, for $\omega>2$,

$$
\mathrm{E}\left(N_{10} \mid 0 \text { before } \omega\right)=1+\left(\frac{2}{1-1 / z_{\omega}}\right) \sum_{r=1}^{\omega-2} \frac{\left(1-z_{r} / z_{\omega}\right)\left(1-z_{r+1} / z_{\omega}\right)}{L_{r+1}} .
$$


It is now simple to find the conditional expected value of $N_{k 0}$ for any $k>0$, given that 0 is reached before $\omega>k$. For we may write $N_{k 0}=N_{k, k-1}+\cdots$ $+N_{10}$ where the terms in the sum are independent. Hence

$$
\mathrm{E}\left(N_{k 0} \mid 0 \text { before } \omega\right)=\sum_{j=0}^{k-1} \mathrm{E}\left(N_{k-j, k-j-1} \mid 0 \text { before } \omega\right) .
$$

Now $\mathrm{E}\left(N_{k-j, k-j-1} \mid 0\right.$ before $\left.\omega\right)=\mathrm{E}\left(N_{k-j, k-j-1} \mid k-j-1\right.$ before $\left.\omega\right)$, because of the Markovian nature of the process. From (4.9), by a translation of indices, we have

$$
\begin{aligned}
& \mathrm{E}\left(N_{j, j-1} \mid j-1 \text { before } \omega\right) \\
& \quad=1+\frac{2 L_{j}}{\left(z_{\omega}-z_{j-1}\right)\left(z_{\omega}-z_{j}\right)} \sum_{r=1}^{\omega-j-1} \frac{\left(z_{\omega}-z_{j-1+r}\right)\left(z_{\omega}-z_{j+r}\right)}{L_{j+r}}=C_{j \omega .}
\end{aligned}
$$

Then $\mathrm{E}\left(N_{k 0} \mid 0\right.$ before $\left.\omega\right)=\sum_{j=0}^{k} C_{j \omega}$, where $C_{j \omega}$ is defined in (4.10).

It is interesting to note that if the $p_{r}$ have the form

$$
p_{r}=\frac{1}{2}+\frac{c}{r}+O\left(\frac{1}{r^{2}}\right)
$$

where $c<-1 / 4$, then, using (4.2), we have

$$
\lim _{k \rightarrow \infty} \theta_{k 0}=0
$$

and, as pointed out before, Theorem 2 is in this case rather devoid of content. On the other hand, if the limit superior of $p_{k} / q_{k}$ is less than 1 as $k \rightarrow \infty$, it is clear that the limit inferior of $\theta_{k 0}$ is positive.

5. Generating functions for random walk variables. It is customary to treat random walk problems by means of linear difference equations. In this section we use a simple nonlinear recurrence relation which seems well adapted to certain random walk problems and which, as far as the author knows, has not been used before.

We give explicit expression for the second moments of typical firstpassage variables. We shall indicate briefly how these can be used to give a more precise form, for random walks, of the exponential limiting results of $\S 3$; Lemma 3 holds for some walks with infinite mean recurrence times.

Theorem 4. Assume either condition $\mathrm{A}$ or $\mathrm{B}(\omega)$ of $\$ 4$ holds. Let $h_{k}(s)$ be the generating function for the random variable $(1 / 2)\left(1+N_{k, k+1}\right)$,

$$
h_{k}(s)=\sum_{j=1}^{\infty} s^{i} P\left(\frac{1+N_{k, k+1}}{2}=j\right) .
$$

Then $h_{k}(s)$ satisfies the recursion formula

$$
h_{k}(s)=s T_{k}\left(h_{k-1}(s)\right), \quad k=1,2, \cdots,
$$


where

$$
T_{k}(u)=p_{k} /\left(1-q_{k} u\right) .
$$

Theorem 4 holds even if $h_{k}(1)<1$; i.e., even if $N_{k, k+1}$ has a positive probability of being infinite.

The relation (5.1) is analogous to certain formulae which appear in the theory of branching stochastic processes. As we shall see in $\S 6$, the resemblance is more than superficial.

To prove (5.1) we first consider the generating function of $N_{k, k+1}$, which we designate by $f_{k}(s)$. In a passage from $k$ to $k+1$ there will first be $r$ occurrences of the following event: the state goes directly from $k$ to $k-1$ and then continues changing until its first arrival back at $k$. After $r$ such occurrences, the state goes directly to $k+1$. For a fixed $r=0,1,2, \cdots$, the generating function for the number of steps required is $s\left(s f_{k-1}(s)\right)^{r}$. Since the probability for a given $r$ is $p_{k}\left(q_{k}\right)^{r}$, we have

$$
f_{k}(s)=\sum_{r=0}^{\infty} p_{k}\left(q_{k}\right)^{r} s\left(s f_{k-1}(s)\right)^{r}=\frac{s p_{k}}{1-q_{k} s f_{k-1}(s)} .
$$

Since $s f_{k}(s)=h_{k}\left(s^{2}\right),(5.1)$ follows from (5.2). There is no difficulty about infinite values of $N_{k, k+1}$, provided we adopt the convention, in the argument just given, that even when $N_{k, k+1}$ is infinite the state does finally go from $k$ to $k+1$ after infinitely many steps.

In the special case of a reflecting barrier at $0, h_{0}(s)=s$ and (5.1) gives a means of obtaining $h_{k}(s)$ for all $k$.

It is clear that the moments of $(1 / 2)\left(1+N_{k, k+1}\right)$ satisfy recursion relations which can be obtained by differentiating (5.1) and putting $s=1$. For example, setting

$$
M_{k}=\frac{1}{2}\left(1+N_{k, k+1}\right)
$$

we have

$$
h_{k}(1)=P\left(M_{k}<\infty\right)=T_{k}\left(h_{k-1}(1)\right) .
$$

In the recurrent case both sides of (5.3) are equal to 1 . Differentiation gives the relations (which we write down only for the recurrent case)

$$
\begin{aligned}
\mathrm{E}\left(M_{k}\right) & =1+\frac{q_{k}}{p_{k}} \mathrm{E}\left(M_{k-1}\right), \\
\mathrm{E}\left(M_{k}^{2}-M_{k}\right) & =\frac{2 q_{k}}{p_{k}} \mathrm{E}\left(M_{k-1}\right) \mathrm{E}\left(M_{k}\right)+\frac{q_{k}}{p_{k}} \mathrm{E}\left(M_{k-1}^{2}-M_{k-1}\right) .
\end{aligned}
$$

For the reflecting barrier case (where $p_{0}=1$ ) we have $M_{0}=(1 / 2)\left(1+N_{01}\right)=1$, and (5.4) gives 


$$
\begin{aligned}
\mathrm{E}\left(M_{k}\right)=\frac{1}{2} \mathrm{E}\left(1+N_{k, k+1}\right)=1 & +\frac{q_{k}}{p_{k}}+\frac{q_{k} q_{k-1}}{p_{k} p_{k-1}}+\cdots \\
& +\frac{q_{k} q_{k-1} \cdots q_{1}}{p_{k} p_{k-1} \cdots p_{1}}, \quad k>0,
\end{aligned}
$$

which agrees, after a transformation, with Theorem 3. Similarly, we can use (5.5) to obtain the variance of $N_{k, k+1}$ in the reflecting barrier case, obtaining

(5.7) Variance $\left(N_{k, k+1}\right)=4\left(b_{k}-b_{k}^{2}\right)+8 \frac{q_{1} \cdots q_{k}}{p_{1} \cdots p_{k}} \sum_{r=1}^{k}\left[\left(\frac{p_{1} \cdots p_{r}}{q_{1} \cdots q_{r}}\right)\left(b_{r}^{2}-b_{r}\right)\right]$ if there is a reflecting barrier at 0 , where

$$
b_{r}=1+\frac{q_{r}}{p_{r}}+\frac{q_{r} q_{r-1}}{p_{r} p_{r-1}}+\cdots+\frac{q_{r} q_{r-1} \cdots q_{1}}{p_{r} p_{r-1} \cdots p_{1}} .
$$

At this point it is convenient to write down an expression derived from (5.7) by a change of indices: if $p_{k}=0$ (reflecting barrier at $k$ ), then

$$
\begin{aligned}
& \text { Variance }\left(N_{10}\right)=-4\left(\sum_{r=1}^{k} \frac{1}{L_{r}}\right)\left(\sum_{r=2}^{k} \frac{1}{L_{r}}\right) \\
& +8 \sum_{r=1}^{k-1}\left\{L_{r}\left(\sum_{j=r}^{k} \frac{1}{L_{j}}\right)\left(\sum_{j=r+1}^{k} \frac{1}{L_{j}}\right)\right\}
\end{aligned}
$$

where $L_{1}=1, L_{r}=q_{1} \cdots q_{r-1} / p_{1} \cdots p_{r-1}, r>1$.

From (5.8) it follows that in any recurrent random walk where condition A of $\$ 4$ holds we have

$$
\begin{aligned}
\operatorname{Variance}\left(N_{10}\right)= & -4\left(\sum_{r=1}^{\infty} \frac{1}{L_{r}}\right)\left(\sum_{r=2}^{\infty} \frac{1}{L_{r}}\right) \\
& +8 \sum_{r=1}^{\infty}\left\{L_{r}\left(\sum_{j=r}^{\infty} \frac{1}{L_{j}}\right)\left(\sum_{j=r+1}^{\infty} \frac{1}{L_{j}}\right)\right\} .
\end{aligned}
$$

We now re-examine the arguments leading to Lemma 3 of $\S 3$.

For simplicity we assume throughout the rest of this section that

$$
\text { Condition C: } p_{0}=1
$$

holds.

In deriving Lemma 3 , the assumption of a finite mean recurrence time enabled us to say that $\left[u / \theta_{0 k}\right]$ cycles from 0 to 0 correspond, with high probability, to almost exactly $u / \pi_{0} \theta_{0 k}$ steps when $k$ is large. In the case of random walks, however, it is clear that the distribution of $N_{0 k}$ does not depend on the value of the $p_{r}$ for $r \geqq k$. Therefore, let us suppose that the original 
random walk is altered by placing a reflecting barrier at $k>0$; since condition $\mathrm{C}$ holds, this will make the recurrence times finite.

The modified random walk will have a new set of stationary probabilities $\pi_{r}(k), r=0,1, \cdots, k$. The question is then whether $\left[u / \theta_{0 k}\right]$ cycles from 0 to 0 in the modified random walk correspond almost exactly to $u /\left(\pi_{0}(k) \theta_{0 k}\right)$ steps. We know that the number of steps in a cycle of the modified walk, having a finite mean value, satisfies the weak law of large numbers, but is $\left[u / \theta_{0 k}\right]$ cycles a "large enough" number to make the sample mean almost equal to the true mean? Clearly it is sufficient to have

$$
\lim _{k \rightarrow \infty}\left(\pi_{0}(k)\right)^{2} \theta_{0 k} \text { Variance }\left(N_{10}(k)\right)=0
$$

where $N_{10}(k)$ is the random variable $N_{10}$ in the modified random walk. To apply (5.10) to any particular case, we can refer to (4.2) and (5.8).

As an example of a case where the mean recurrence times are infinite but (5.10) still holds, suppose $p_{r}=1 / 2-1 / 4 r, r=1,2, \cdots$. From Theorems 2 and 3 this corresponds to a recurrent random walk with infinite mean recurrence times. Then

$$
L_{r}=\frac{q_{1} q_{2} \cdots q_{r-1}}{p_{1} p_{2} \cdots p_{r-1}} \sim c_{1} r, \quad r \rightarrow \infty,
$$

where $c_{1}$ is independent of $r$, and an easy calculation shows that (5.10) holds.

Although we shall not enter into details here, the results of this section can be used to give error terms for Theorem 1 or Lemma 3 in the case of random walks with finitely many states. In particular, some of the results of [1] for the time-continuous Ehrenfest model can be obtained for the usual Ehrenfest model with a discrete time parameter. The argument is simple but tedious, depending on the use of Chebyshev's inequality.

6. Walks and trees. $\left({ }^{4}\right)$ Random walks and branching processes are both objects of considerable interest in probability theory. We may consider a random walk as a probability measure on sequences of steps-that is, on "walks," as defined below. A branching process is a probability measure on "trees," as defined below. The purpose of the present section is to show that walks and trees are abstractly identical objects and to give probabilistic consequences of this correspondence. The identity referred to is nonprobabilistic and is quite distinct from the fact that a branching process, as a Markov process, may be considered in a certain sense to be a random walk, and also dis-

(4) I. J. Good has pointed out the similarity between certain formulae in branching processes and random walks [5]. Mr. Good has also informed me by letter that D. G. Kendall has recently shown a relationship between branching processes and the theory of queues, and Good himself has shown a connection between the theory of queues and the gambler's ruin problem [7]. 
tinct from the fact that each step of the random walk, having two possible directions, represents a two-fold branching.

By a "walk" we shall mean any finite sequence of integers $n_{0}, n_{1}, \cdots, n_{r}$ satisfying the following conditions:

$$
\begin{array}{lr}
n_{0}=n_{r}=0, & \\
\quad n_{j}>0, & 1 \leqq j \leqq r-1, \\
\left|n_{j}-n_{j+1}\right|=1, & j=0,1, \cdots, r-1 .
\end{array}
$$

Notice that our walks begin at 0 and terminate as soon as 0 is reached, and we consider for the time being only those which do come back to 0 .

By a "tree" we shall mean a finite set of objects having the relationships of a (male) family tree descended from a single ancestor. A typical element of the tree can be designated by a symbol of the form

$$
z_{m_{1} m_{2} \cdots m_{p}} \text {, }
$$

meaning the $m_{p}$ th son of the $\cdots$ of the $m_{2}$ th son of the $m_{1}$ th son of the original ancestor. For our purpose the two trees

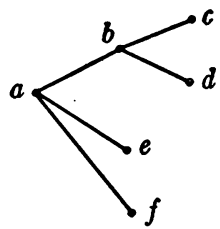

and

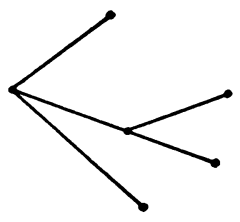

are distinct objects, since we keep track of the "order of birth" of the sons of a given father.

To exhibit the correspondence, we lay down the general principle that a step to the right in the walk, say, from $k$ to $k+1$, corresponds to a birth of an object in the $k$ th generation. The "parent" of this step is the last preceding step from $k-1$ to $k$. The "children" of a given step from $k$ to $k+1$ (let us call this step $S$ ) are the steps from $k+1$ to $k+2$ which occur after $S$, but before any step from $k+1$ to $k$ succeeding $S$. The "children" are numbered in the order of appearance-the first step is oldest, etc.

A step to the left, say, from $k+1$ to $k$, means that the person corresponding to the last preceding step from $k$ to $k+1$ has died and will have no further issue.

Rather than giving a tedious formal demonstration of the correspondence, we shall here only illustrate it. The reader can easily convince himself by working through a few such examples. Consider then the leftmost of the two trees in (6.2). This corresponds to the walk whose successive positions are $0,1,2,3,2,3,2,1,2,1,2,1,0$, if we adopt the convention that of two vertices in the same generation pictured in the tree, the upper is the elder. 
The correspondence, step by step, is as follows $((0,1)$ means a step from 0 to 1 , etc.): $(0,1) \leftrightarrow a$ appears; $(1,2) \leftrightarrow b$ is born; $(2,3) \leftrightarrow c$ is born: $(3,2) \leftrightarrow c$ dies; $(2,3) \leftrightarrow d$ is born; $(3,2) \leftrightarrow d$ dies; $(2,1) \leftrightarrow b$ dies; $(1,2) \leftrightarrow e$ is born; $(2,1) \leftrightarrow e$ dies; $(1,2) \leftrightarrow f$ is born; $(2,1) \leftrightarrow f$ dies; $(1,0) \leftrightarrow a$ dies. Similarly, the other tree in (6.2) corresponds to the walk $0,1,2,1,2,3,2,3,2,1,2,1,0$.

Now suppose that probabilities $p_{k}, q_{k}=1-p_{k}$ are prescribed for the random walk, with $0<p_{k}<1, k=1,2, \cdots$. Consider next the following branching process: an individual in the $(k-1)$ st generation has a probability $p_{k}^{r} q_{k}$ of having exactly $r$ children, $r=0,1, \cdots$. (The ancestor is the 0 generation.) Then we have the following result:

THEOREM 5. The probability that a random particle starting at 1 reaches 0 for the first time after $2 r-1$ steps equals the probability that the family tree just defined becomes extinct after producing a total of $r$ individuals, $r=1,2, \cdots$. The total probability that the particle returns to 0 equals the probability that the family tree is finite.

The proof, which we omit, follows from the identity between walks and trees previously demonstrated; it can also be shown by the use of generating functions.

As an example we consider the classical case $p_{k}=p$. The expected number of children of a single individual in the family tree is $q \sum_{r=0}^{\infty} r p^{r}=p /(1-p)$. If $p \leqq 1 / 2$, i.e., $p /(1-p) \leqq 1$, the total number of individuals in the tree is finite with probability 1 , and the generating function $\phi(s)$ of the total number satisfies the relation

$$
\phi(s)=\frac{s q}{1-p \phi(s)},
$$

whence we have (see Otter [9], or Hawkins and Ulam [6])

$$
\phi(s)=\frac{1-(1-4 p q s)^{1 / 2}}{2 p} .
$$

It can be verified that $\phi(s)$ is likewise the generating function for the random variable $(1 / 2)\left(1+N_{10}\right)$. In the case $p>1 / 2, \phi(s)$ has the same form, but in this case $\phi(1)=q / p$; this is the probability that the family is finite and, as is well known, is likewise the probability that $N_{10}$ is finite.

If condition $\mathrm{B}(\omega)$ holds, the generating function of the random variable $(1 / 2)\left(1+N_{10}\right)$ can be obtained by changing the numbering system of the lattice points in Theorem 4 . We obtain

$$
\sum_{r=0}^{\infty} s^{r} P\left(\frac{1+N_{10}}{2}=r\right)=s T_{1}^{*}\left(s T_{2}^{*}\left(\cdots\left(s T_{\omega-1}^{*}(s)\right) \cdots\right)\right)
$$

where $T_{r}^{*}(u)=q_{r} /\left(1-p_{r} u\right)$. This formula is likewise the generating function 
for the total number of individuals in generations 0 through $\omega-1$ of the family tree. The generating function for $(1 / 2)\left(1+N_{10}\right)$ in the case where condition $\mathrm{A}$ holds (and for the total number of individuals in the family tree) is obtained by letting $\omega$ go to $\infty$ in (6.3).

\section{REFERENCES}

1. R. Bellman and T. Harris, Recurrence times for the Ehrenfest model, Pacific Journal of Mathematics vol. 1 (1951) pp. 184, 187.

2. K. L. Chung, Contribution to the theory of Markov chains, Journal of Research, National Bureau of Standards (to appear).

3. W. Doeblin, Sur deux problèmes de M. Kolmogoroff concernant les chaînes dénombrables, Bull. Soc. Math. France vol. 52 (1942) pp. 37-64.

4. W. Feller, Probability theory and its applications, Wiley, 1950.

5. I. J. Good, Random motion on a finite Abelian group, Proc. Cambridge Philos. Soc. vol. 47 (1951) p. 761.

6. D. Hawkins and S. Ulam, Theory of multiplicative processes, I, (1944), Los Alamos Declassified Document 265.

7. D. G. Kendall, Some problems in the theory of queues, Journal of the Royal Statistical Society, Ser. B, vol, 13 (1951) pp. 151-185.

8. Paul Lévy, Systèmes markoviens et stationnaires, cas dénombrables, Ann. École Norm. (to appear).

9. R. Otter, The multiplicative process, Ann. Math. Statist. vol. 20 (1949) pp. $212 \mathrm{ff}$.

10. A. Wald, Sequential analysis, Wiley, 1947, pp. 52-53.

The Rand Corporation,

Santa Monica, Calif. 